\title{
GUIDING SCHEMA INTEGRATION BY BEHAVIOURAL INFORMATION
}

\author{
Christiaan Thieme and Arno Siebes
}

CWI, P.O. Box 94079, 1090 GB Amsterdam, The Netherlands

(Received August 1994; in final revised form 10 April 1995)

\begin{abstract}
This paper presents an approach to schema integration, where schemas are restructured using schema transformations and schemas are merged using join operators. The novelty of the approach is that behavioural information is used to guide schema restructuring as well as schema merging.
\end{abstract}

Key words: Schema Integration, Behaviour, Object Oriented Databases

\section{INTRODUCTION}

Schema integration is an important and non-trivial task in database design. It occurs when a number of different user views, developed for a new database system, or a number of existing database schemas must be integrated into a global, unified schema. As schema integration is a diffcult task, methods to support the designer with this task are essential. In [6], a framework for comparing integration methods is given. The framework idertifies four steps. In the first step, the preintegration step, an integration strategy is chosen and additional information on the schemas is gathered. Subsequently, the schemas are analysed and compared to find similarities/conflicts among the schemas. In the conforming step, the condicts found in the comparison step have to be resolved. Finally, in the last step, the schemas are merged by superimposition and the resulting schema is analysed and restructured if necessary.

For our purpose, the main characteristic of an integration method is: which similarities/conflicts are detected and how are conflicts resolved? A number of integration methods use assertions among different component schemas to compare attributes and entity types. In [17], interschema assertions, names, and types are used to compare object types. In [15], schemas are merged using schema operators and assertions among entity types and attributes ir diferent schemas. And in [13], attribute assertions (e.g., key/non-key and lower/upper bounds) are used to compare attributes and entity types. However, the assertions must be supplied by the designer and the resolution of conflicts depends heavily on the common sense of the designer. Other methods use schema transformations to resolve structural conflicts. In [8], structural transformations are defined to integrate compatible structures. In [16], a number of schema transformations (e.g., join and meet) are proposed to restructure schemas. And in [5], transformations between attributes, entity types, and relationship types are used to resolve type conflicts. However, only the last one gives a heuristic (viz., concept likeness/unlikeness) for applying the transformations. Finally, a number of recent methods use more specific information on semantical properties of attributes and entity types to detect similarities and conflicts. In [18, 23], attribute assertions are used to define relationships between an attribute on one hand and a semantic point or a set of concepts on the other hand. Again, the assertions must be supplied by the designer. In [19], a database metadictionary is used to define a semantic domain for each attribute. And in [9], a terminological knowledge base containing information on negative and positive associations between terms and information on specialisation of terms is used to compare entity types.

This paper presents a new approach to schema integration, based on schema transformations and the approach taken in $[20,21]$, where classes are compared by structure and by behaviour. The approach consist of two steps. First, component schemas are restructured using schema iransformations, and syntactical properties of methods are used to guide the restructuring process. Subsequently, the component schemas are merged using join operators, and semantical properties 
of methods are used to guide the merging process. More details on the approach are given in [22]. There is, as far as the authors know, no other approach that uses methods to compare attributes. For sake of completeness, it should be mentioned that there is an approach to schema evolution that analyses methods ([7]), not to compare attributes, but to solve non-legitimate overriding of methods.

Since behavioural information plays an important role in our method, we have chosen an objectoriented model as our vehicle. In principle, the approach can also be used in a relational or E-R setting, where the behavioural information is hidden in the applications. In practice, however, extracting such information from applications will be much more difficult than extracting it from the object-oriented schema.

Finally, the theoretical basis of our approach is invariant with respect to renaming of classes, attributes and methods. This means that our approach is supplementary to an approach that uses names to find similarities, such as a lexicon-based approach.

The outline of this paper is as follows. In the next section, we give a brief overview and formalisation of our daba model. In Section 3, we define a number of well-known type transformations and extend them to be applicable to recursive types as well. Furthermore, we show how these type transformations induce schema transformations. In Section 4 , we show how methods can be used to guide schema restructuring and give a heuristic algorithm to restructure and merge schemas. In the last section, we summarise and give some directions for further research.

\section{DATABASE SCHEMAS}

In this section, we introduce a subset of the database schemas found in object-oriented database languages such as Galileo [2], Goblin [12], $\mathrm{O}_{2}$ [14], and TM/FM [4].

Informally, an object-oriented database schema is a a class hicrarchy, i.e., a set of classes related by a subclass relation. A class has a name, a set of superclasses, a set of attributes, a set of constraints, and a set of update and query methods.

Definition 1 (Class hierarchies) First, five disjoint sets are postulated: a set $C N$ of class names, a set $A N$ of attribute names, a set $M N$ of method names, a set $L$ of labels, and a set Cons of basic constants (i.e., 'integer', 'rational', and 'string' constants). The sets are generated by the nonterminals $C N, A N, M N, L$, and Cons, respectively. Class hierarchies are the sentences of the following BNF-grammar, where the plus sign $\left({ }^{+}\right)$denotes a finite, nonempty repetition, square brackets (I]) denote an option, and the vertical bar (1) denotes a choice:

\begin{tabular}{|c|c|c|}
\hline Hierarchy & $::=$ & Class $^{+}$ \\
\hline Class & $::=$ & $\begin{array}{l}\text { 'Class' CN ['Isa' CN } \\
{[\text { ['Attributes' Att'] }} \\
{[\text { 'Constraints' Key' }} \\
{[\text { [Methods' Meth }} \\
\text { 'Endclass' }\end{array}$ \\
\hline Att & $::=$ & AN ':' Type \\
\hline Type & $::=$ & BasicType / SetType / RecordType / CN \\
\hline Basic Type & $::=$ & 'integer' | 'rational' | 'string' \\
\hline SetType & $::=$ & ' $\{$ ' Type ' $\}$ ' \\
\hline RecordType & $::=$ & '<'FieldList ' $\rangle$ ' \\
\hline FieldList & $::=$ & Field | Field ',' FieldList \\
\hline Field & $::=$ & L :' Type \\
\hline Key & $::=$ & key KeyAtt ${ }^{+}$ \\
\hline KeyAtt & $::=$ & AN $\mid$ KeyAtt $\because \mathrm{L}$ \\
\hline Meth & $::=$ &  \\
\hline ParList & $::=$ & Par | Par "'ParList \\
\hline Par & $::=$ & L :' BasicType \\
\hline Result & $::=$ & L :' Type \\
\hline
\end{tabular}




\begin{tabular}{|c|c|c|}
\hline AsnList & $::=$ & Assign | Assign ';' AsnList \\
\hline Assign & $::=$ & Dest ':=' Source | 'insert(' Source ',' Dest ')' \\
\hline Dest & $::=$ & L | AN | L' 'Dest | AN '? Dest \\
\hline Source & $::=$ & $\begin{array}{l}\text { 'self' | Term | Term '+' Source | Term '-' Source | } \\
\text { Term ' } X \text { ' Source | Term ' } \div \text { ' Source | 'new (' CN ')' | } \\
\text { Dest ' ' MN '(' ActParList ')' }\end{array}$ \\
\hline Term & $::=$ & Dest | Cons \\
\hline ActParList & $::=$ & Term | Term "' ActParList \\
\hline
\end{tabular}

A class hierarchy is well-defined if it satisfies four conditions. The first condition is that the Isa relation is acyclic, and classes have a unique name and only refer to classes in the class hierarchy. The second is that attributes have a unique name within their class and are well-typed. The third is that keys must be well-defined. The fourth is that methods have a unique name within their class and are well-typed.

\subsection{Underlying types}

Informally, the set of all attributes of a class consists of both the new and inherited attributes.

Definition 2 (Attributes) Let $H$ be a class hierarchy satisfying the first condition for weildefined class hierarchies. We abbreviate every class in $H A$ to a 5 -tuple $(c, S, A, K, M)$, where $c$ is the name of the class, $S$ is the set of (names of) superclasses, $A$ is the set of new attributes, $K$ is the set of new keys, and $M$ is the set of new methods. Now let $C=(c, S, A, K, M)$ be an abbreviated class in $H$. The name of $C$ is denoted by name( $C$ ) and the set of all attributes of $C$, denoted by atts $(C)$, is defined as:

$$
\operatorname{atts}(C)=A \cup\left\{a: T \mid \exists C^{\prime} \in H:\left[\text { name }\left(C^{\prime}\right) \in S \wedge a: T \in a t t s\left(C^{\prime}\right) \wedge \forall a^{\prime}: T^{\prime} \in A\left[a \neq a^{\prime}\right]\right\} .\right.
$$

Since we require that the Isa relation is acyclic, atts is well-defined.

Every class in a class hierarchy has an underiying type, which describes the structure of the class, i.e., the structure of the objects in its extensions (cf. TM/FM [4]). The underlying type of a class is an aggregation of its attributes, where recursive types [3] are used to cope with attributes that refer to classes.

Definition 3 (Underlying types) First, postulate a new type 'oid', whose extension is an enumerable set of object identifiers. Let $H$ be a class hierarchy satisfying the first condition, $C$ be a class in $H$, and $c$ be the name of $C$. The underlying type of class $C$, denoted by type $(C)$, is defined as:

$$
\text { type }(C)=\tau(c, D)
$$

where

$$
\begin{aligned}
& \tau(d, V)=\mu d .<\text { id }: \text { oid, } a_{1}: \tau\left(T_{1}, V \cup\{d\}\right), \cdots, a_{k}: \tau\left(T_{k}, V \cup\{d\}\right)> \\
& \quad \text { if } d \notin V \text { and } \exists D \in H\left[\text { name }(D)=d \wedge \text { atts }(D)=\left\{a_{1}: T_{1}, \cdots, a_{k}: T_{k}\right\}\right], \\
& \tau(d, V)=d \text { if } d \in V, \\
& \tau(B, V)=B \text { if } B \in\{\text { integer, rational, string }\}, \\
& \tau(\{U\}, V)=\{\tau(U, V)\}, \\
& \tau\left(<l_{1}: U_{1}, \cdots, l_{n}: U_{n}>, V\right)=\left\langle l_{1}: \tau\left(U_{1}, V\right), \cdots, l_{n}: \tau\left(U_{n}, V\right)>.\right.
\end{aligned}
$$

Set $V$ contains the names of the classes for which a (recursive) type is being constructed as part of the construction of the underlying type of class $C$. If $V$ contains $d$, then $\tau(d, V)=d$ indicates a repetition of the recursive type.

The set of all types, denoted by Types, is defined as the co-domain of $\tau$, i.e., it consists of all types that can be constructed using $\tau$.

Note that the underlying type of a class depends on the hierarchy. 


\section{SCHEMA TRANSFORMATIONS}

In this section, we give an overview of type transformations and show how type transformations induce schema transformations.

The set of basic type transformations consists of renaming, aggregation, and objectification (cf. [1] and [10]).

Definition 4 (Easic type transformations) Let $\mathcal{L}$ be the union of $L$ and $A N$ and Types be the set of types introduced in Definition 3. Renaming is defined as a function of type $(\mathcal{L}-\{$ id $\}) \rightarrow$ $(\mathcal{L}-(\mathrm{id}) \rightarrow$ Types $\rightarrow$ Iypes:



Note that we do not allow renaming of id-fields. Aggregation is defined as a function of type $\wp(\mathcal{L}) \rightarrow(\mathcal{L}-\{$ id $\}) \rightarrow$ Types $\rightarrow$ Types:



For all other cases, we have: aggregate $(L)(l)(v)=v$. Objectification is defined as a function of type Types $\rightarrow$ Types:

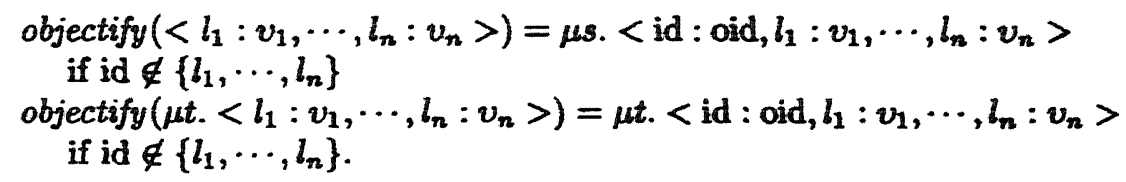

For all other cases, we have: $\operatorname{objectify}(v)=v$.

Complex type transformations are obtained by combining basic type transformations.

Example 1 Type $\sigma=\left\langle l_{1}: \mu s\right.$. < id : oid, $l: v_{1}, l_{2}: v_{2}>, l_{3}: v_{3}>$ can be obtained from type $\sigma_{1}=\left\langle l_{1}: v_{1}, l_{2}: v_{2}, l_{3}: v_{3}\right\rangle$ as follows:

$$
\begin{aligned}
& \sigma_{2}=\text { rename }\left(l_{1}\right)(l)\left(\sigma_{1}\right)=\left\langle l: v_{1}, l_{2}: v_{2}, l_{3}: v_{3}\right\rangle \\
& \sigma_{3}=\text { aggregate }\left(\left\{l_{3}, l_{2}\right\}\right)\left(l_{1}\right)\left(\sigma_{2}\right)=\left\langle l_{1}:\left\langle l: v_{1}, l_{2}: v_{2}\right\rangle, l_{3}: v_{3}\right\rangle \\
& \left.\sigma_{4}=\left\langle l_{1}: \text { objectify }\left(<l: v_{1}, l_{2}: v_{2}\right\rangle\right), l_{3}: v_{3}\right\rangle=\sigma .
\end{aligned}
$$

Both the transformation for lexical attributes and the transformation for unstable subtypes from [11] can be obtained by composing one aggregate and one objectify operation.

Example 2 The following class hierarchy introduces a class Person, a class Employee, which inherits from class Person, and a class Company: 


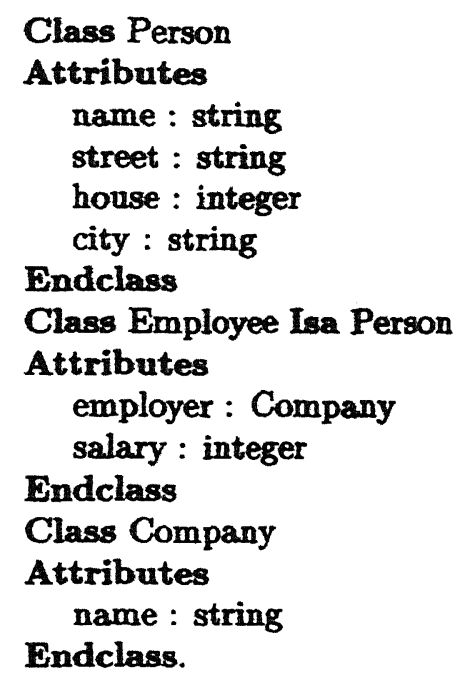

The underlying type of class Person is:

$\mu$ Person. <id:oid, name:string, street:string, house:integer, city:string>.

The underlying type of class Person can be transformed into (by applying aggregate (\{street,house, city\}) (address)):

$\mu$ Person. <id:oid, name:string, address:<street:string, house:integer, city:string>>,

which can be transformed into (by applying objectify to the type of address):

$\mu$ Person. <id:oid, name:string, address: $\mu$ Address. <id: oid, street:string, house:integer, city:string>>

The composite transformation is a variant of the transformation for lexical attributes from [11]. We can redefine class Person as a class (named Person1) that refers to a new class (named Address):



The underlying type of class Employee is:

$\mu$ Employee. <id:oid, name:string, street:string, house:integer, city:string, employer: $\tau_{C}$, salary:integer $>$,

where $\tau_{C}$ is the underlying type of class Company. The underlying type of class Employee can be transformed into (by applying aggregate (\{id,name,street,house,city $\}$ ) (employee)):

$\mu$ Works for. <employee : $\mu$ Employee. <id:oid, namesstring, street:string, house:integer, city:string $>$, employer: $\tau_{C}$, salary:integer $>$,

which can be transformed into (by applying objectify): 
$\mu$ Works_for. <id:oid, employee : $\mu$ Employee. <id:oid, name:string, street:string, house:integer, city:string $>$, employer: $T_{C}$, salary:integer $>$.

The composite transformation is a variant of the transformation for unstable subtypes from [11]. We can redefine class Employee as a 'relation' (named Works_for) that refers to a new class (named Employee1):



Note that, in the original situation, an employee (an object in class Employee), does have a unique employer, whereas, in the resulting situation, an employee (an object in class Employeel) does not. Therefore, we define a key for class Worksfor:

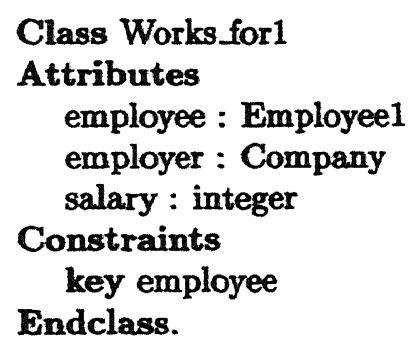

\section{APPLICATION OF SCHEMA TRANSFORMATIONS}

In the previous section, we defined type transformations and showed how they induce schema transformations. In this section, we show how behaviour of methods can be used to choose among a set of schema transformations.

A class can be transformed in several ways, using different factors and different transformations.

Example 3 Let class Employee be the following class:

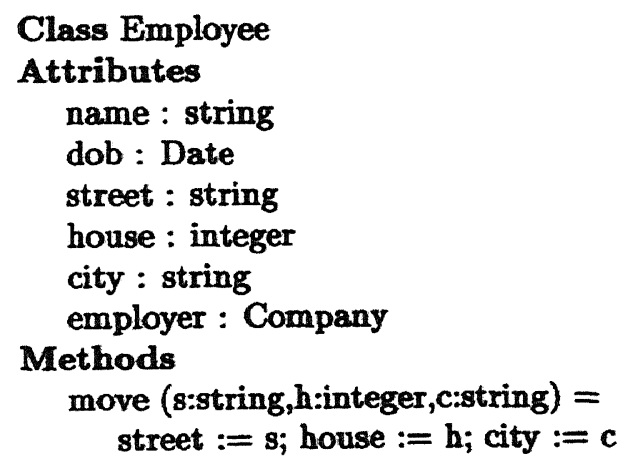

\section{Endclass}

and class Address be a factor of Employee: 




\section{Endclass.}

One option to transform class Employee is to redefine Employee as a subclass of Address (factorisation by specialisation):

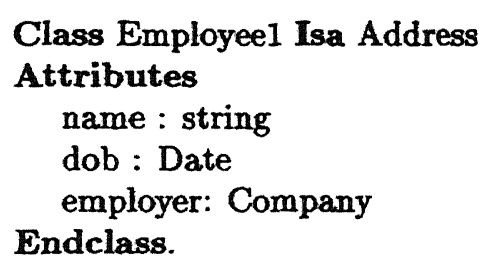

Another option is to redefine Employee as a class referring to Address (factorisation by delegation):

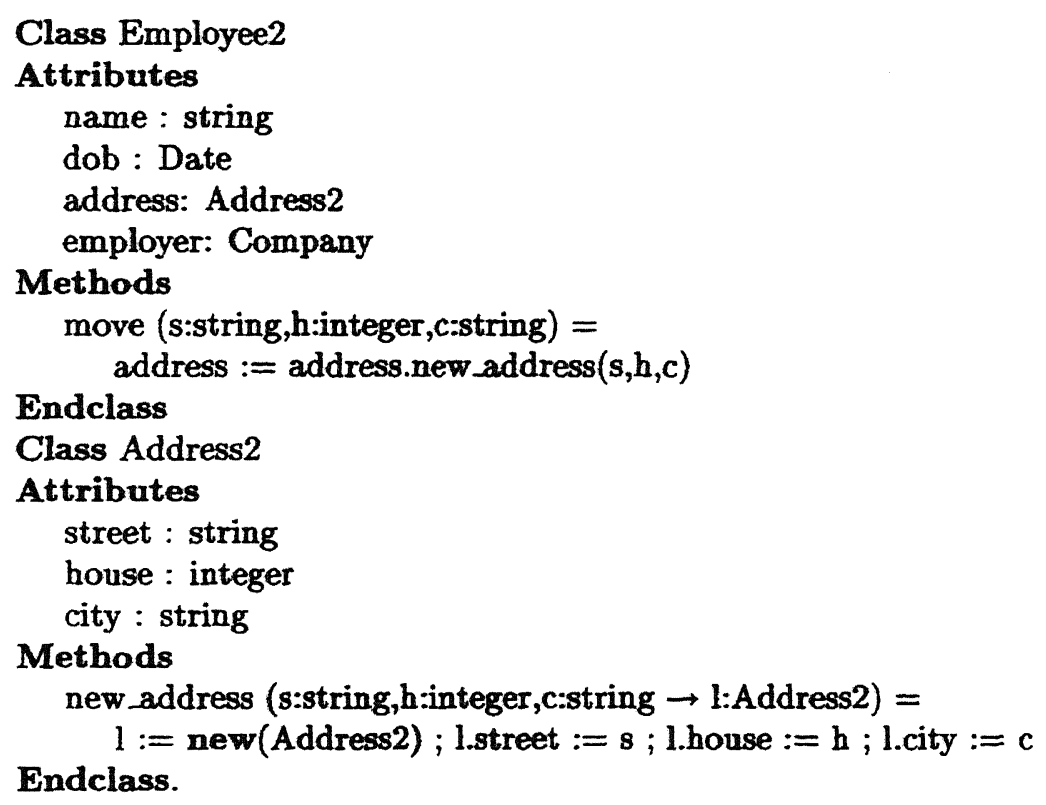

Note that, as an employee is not an address in the real world, it is unlikely that the first option is the right choice. The second option, where employee refers to an address (as one of its attributes) is a more reasonable choice. Now, let class Person be a factor of class Employee2:

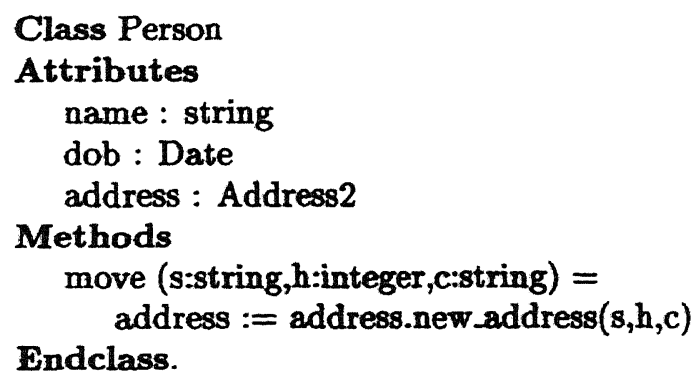

One option to transform class Employee2 is to redefine Employee2 as a subclass of Person (factorisation by specialisation): 
Class Employee3 Isa Person

Attributes employer : Company

Endclass.

Another option is to redefine Employee2 as a class referring to Person (factorisation by delegation):

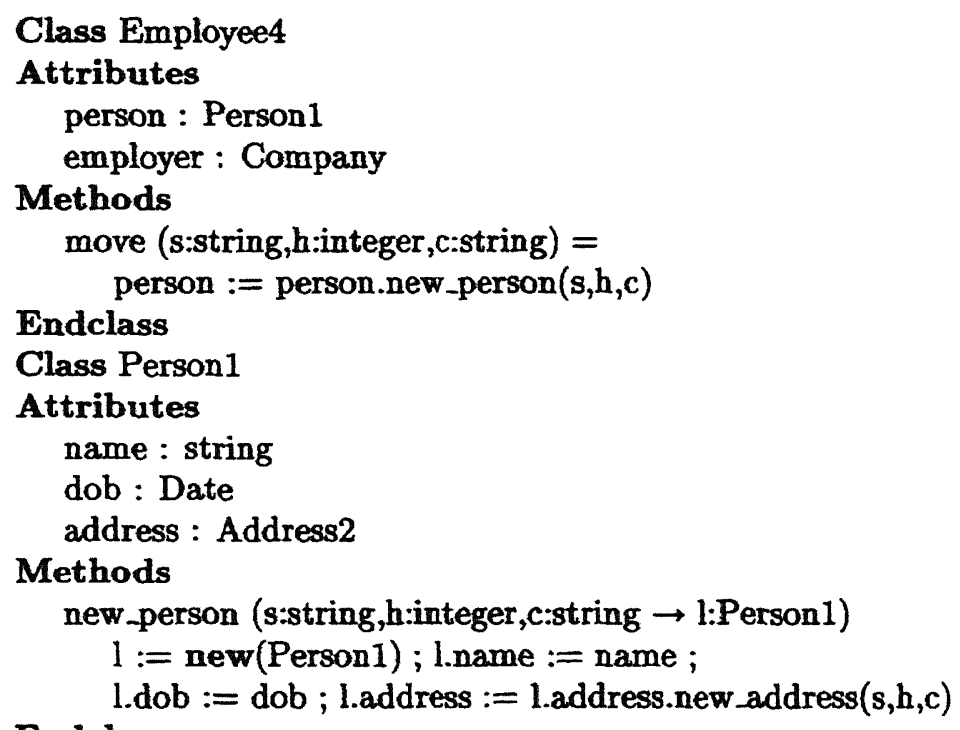

\section{Endclass.}

Since the objects in class Employee2 become the objects in class Employee4, we redefine method 'move' to be applicable to objects in class Employee4. Yet another option is to redefine class Employee2 as a relation involving class Person:



Since the objects in class Employee2 become the objects in class Person, we do not redefine method 'move', because it is already applicable to objects in class Person.

Note that, as an employee is a person in the real world, it is likely that options one and three are more reasonable than option two, where an employer refers to a person (as one of its attributes).

As we have seen, a class can be transformed in several ways, using different factors and different transformations, e.g., factorisation by specialisation, factorisation by delegation, or redefinition as a relation. But how do we choose factors and how do we choose between specialisation, delegation and redefinition as a relation? For that purpose, we introduce evidence ratios for relatedness. Weak relatedness for a set of attributes says whether the attributes are mutually related (according to the methods). Strong relatedness for a set of attributes says whether the attributes are mutually related, but not to attributes outside the set (according to the methods). Isolation for a set of attributes says whether the attributes are not related to attributes outside the set (according to the methods).

Definition 5 (Relatedness ratios) Let $H$ be a well-defined class hierarchy, $C$ be a class in $H$, $c$ be the name of $C$, and $M$ be the set of all methods of $C$. Furthermore, for meth $\in M$, let atts (meth) consist of the names of attributes of $C$ that occur in meth. Weak relatedness of a set 
of attributes $A \subseteq\{a \mid a: T \in$ atts $(C)\}$ is defined as:

$$
\text { weakrel }(c, A)=\frac{\mid\{\text { meth } \in M \mid \text { atts }(\text { meth }) \supseteq A\} \mid}{\mid\{\text { meth } \in M \mid \text { atts }(\text { meth }) \cap A \neq \emptyset\} \mid} .
$$

Strong relatedness of a set of attributes $A$ is defined as:

$$
\text { strongrel }(c, A)=\frac{\mid\{\text { meth } \in M \mid \text { atts }(\text { meth })=A\} \mid}{\mid\{\text { meth } \in M \mid \text { atts }(\text { meth }) \cap A \neq \emptyset\} \mid} .
$$

Isolation of a set of attributes $A \subseteq\{a \mid a: T \in a t t s(C)\}$ is defined as:

$$
\text { isolation }(c, A)=\frac{\mid\{\text { meth } \in M \mid \text { atts }(\text { meth }) \neq \emptyset \wedge \text { atts }(\text { meth }) \subseteq A\} \mid}{\mid\{\text { meth } \in M \mid \text { atts }(\text { meth }) \cap A \neq \emptyset\} \mid} \text {. }
$$

If $\{$ meth $\in M \mid$ atts (meth) $\cap A \neq \emptyset\}$ is empty, then weakrel $(c, A)$ and strongrel $(c, A)$ are defined to be 0 , and isolation $(c, A)$ is defined to be 1 .

For a set of attributes with strong relatedness ratio 1 and any method, either all attributes occur in the method and all attributes that occur in the method are in the set, or no attribute in the set occurs in the method. In that case, the attributes are strongly related. For a set of attributes with weak relatedness ratio 0 , there is no method in which all attributes occur and, hence, the attributes are not (mutually) related. And for a set of attributes with isolation ratio 1 and any method, either all attributes that occur in the method are attributes in the set or no attribute that occurs in the method is an attribute in the set. In that case, the attributes are only related within the set.

Weak and strong relatedness can help to choose a factor. If the strong relatedness ratio of a set of attributes is high, then it is reasonable to believe that they belong together and, hence, to factorise. On the other hand, if the weak relatedness ratio is low, then it reasonable to believe that they do not belong together and, hence, not to factorise.

Example 4 Consider class Employee of Example 3. The weak and strong relatedness ratios for \{street, house, city $\}$ and $\{$ name, dob $\}$ are given by:

$$
\begin{aligned}
& \text { strongrel }(\text { Employee, }\{\text { street, house, city }\})=1 \\
& \text { weakrel }(\text { Employee, }\{\text { street, house, city }\})=1 \\
& \text { strongrel }(\text { Employee, }\{\text { name, dob }\})=0 \\
& \text { weakrel }(\text { Employee, }\{\text { name, dob }\})=0 .
\end{aligned}
$$

As we can see, street, house, and city are strongly related, whereas name and dob are not related.

Now, consider class Employee2 of Example 3. The weak and strong relatedness ratios for \{name, dob, address\} and \{name, dob, employer\} are given by:

$$
\begin{aligned}
& \text { strongrel (Employee2, }\{\text { name, dob, address }\})=0 \\
& \text { weakrel }(\text { Employee2, } \text { name, dob, address }\})=0 \\
& \text { strongrel }(\text { Employee2, \{name, dob, employer }\})=0 \\
& \text { weakrel (Employee2, } \text { name, dob, employer }\})=0 .
\end{aligned}
$$

As we can see, in both cases the attributes are not related.

Jsolation can help to choose between specialisation and redefinition as a relation. If the isolation ratio is less than one, then specialisation is possible, but redefinition as a relation is not, since, in that case, we have to add a method to the relation that updates another relation or class.

Example 5 Consider class Employee2 of Example 3. The isolation evidence ratio for name, dob, address is given by:

$$
\text { isolation (Employee2, }\{\text { name, dob, address }\})=1 .
$$


Redefinition as a relation results in a relation (Employment) that represents a simple association between a person and a company. Now, if we add a method to class Employee2 that updates attribute address and attribute employer, then we will have to add a method to Employment that creates a new person and updates attribute employee and attribute employer. Since this method is not a simple insert or update operation on Employment, Employment is no longer a relation.

So, how do we choose factors and transformations? Factors are chosen by comparing weak evidence ratios. If the weak evidence ratio of a set of attributes is greater than some threshold, there is reason to assume that the attributes can be used as a factor. If not, there is no reason. Transformations are chosen by comparing strong evidence ratios and isolation ratios. In case the strong evidence ratio is greater than some threshold, delegation is a reasonable option, because the attributes are strongly related within the set and weakly related with other attributes. In case the isolation ratio is less than one, then specialisation is possible, but redefinition as a relation is not. Otherwise, specialisation or redefinition as a relation are both possible. It should be mentioned that, in the context of schema integration, schema transformations must be applied carefully and only if necessary. In particular, this is true for factorisation by specialisation, since a lot of new classes will be generated by this type of transformation.

The considerations for choosing factors and transformations can be used in a heuristic algorithm to support schema integration. First, the attributes of every class are partitioned in such a way that the isolation ratio of every element in the partition is one, and every class is factorised by delegation if desirable. Subsequently, for every pair of promising classes, a set of possible superclasses is computed, and both classes are factorised by specialisation or redefined as a relation if desirable.

Algorithm 1 The following algorithm is a heuristic for integrating two database schemas (resp., DBS1 and DBS2), given thresholds for strong relatedness and weak relatedness (resp., TSR and TWR):

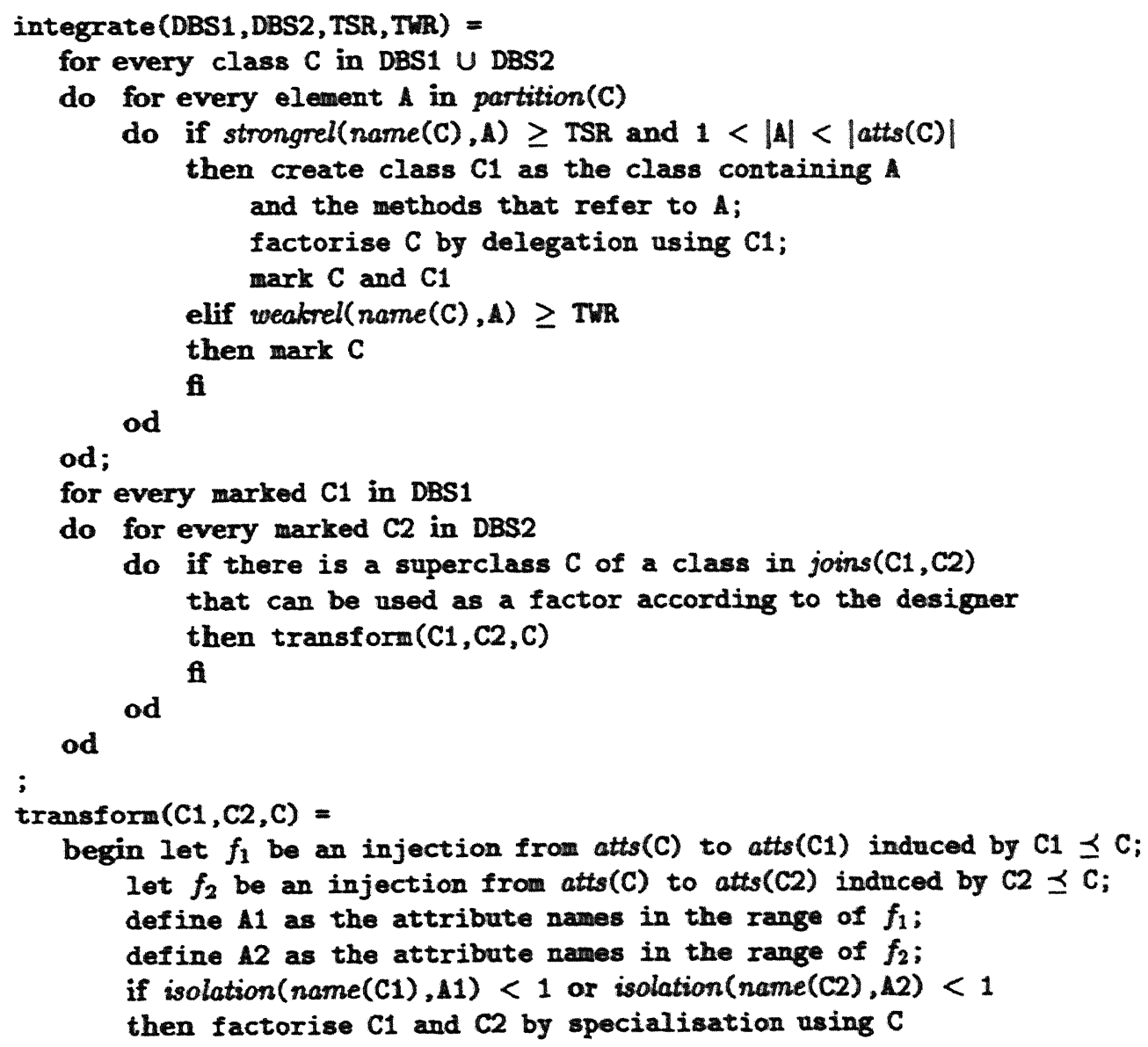






and joins(D1,D2) is the set of common superclasses of D1 and D2, and $\preceq$ is the subclass relation as defined in [21].

Note that the algorithm interacts with the designer. It should be mentioned again that the algorithm is a heuristic and should therefore be used in close interaction with the designer. The heuristic can be improved by combining the different thresholds and refining the different actions. This is the subject of future research.

\section{CONCLUSION}

In this paper, we presented a new approach to schema integration based on transformations and behaviour, because we think that this reflects the importance of behaviour for the real world semantics of objects. First, we formalised schemas using underlying types and underlying constraints. Next, we presented a number of type transformations on underlying types and used them to transform schemas. Finally, we gave a heuristic algorithm for integrating schemas. The algorithm uses schema transformations to restructure schemas and join operators to merge them and behavioural information to guide restructuring and merging.

Our approach differs from other approaches on a number of points:

1. Names. The theoretical basis of our approach is invariant with respect to renaming of classes, attributes, and methods. This means that our approach is supplementary to an approach that uses names to find similarities, e.g., a lexicon-based approach.

2. Structure. We propose using schema transformations already in the comparison phase to detect transformational similarities between classes. This means that more (and more complex) similarities can be detected before interaction with the designer.

3. Behaviour. We also propose using semantic properties of methods to detect similarities between classes and syntactic properties of methods to reduce the amount of work.

Further research includes generalisation and improvement of the theoretical framework, such as extension of the data model and refinement of the heuristic algorithm. Further research also includes construction of an integration tool based on the theoretical framework and practical validation on real world applications using the tool.

Acknowledgements - We wish to thank the anonymous referees for their constructive criticism. This research was partly funded by The Netherlands Organization for Scientific Research (NWO) through NFI-grant NF74. 


\section{REFERENCES}

[1] S. Abiteboul and R. Hull. Restructuring hierarchical database objects. Theoretical Computer Science, 62:3-38 (1988).

[2] A. Albano, L. Cardelli, and R. Orsini. Galileo: A strongly typed, interactive conceptual language. ACM Trans. on Database Systems, 10(2):230-260 (1985).

[3] R. Amadio and L. Cardelli. Subtyping recursive types. In Proc. Int. Symp. on Principles of Programming Langrages, pp. 104-118 (1991).

[4] P. Apers, H. Balsters, R. de By, and C. de Vreeze. Inheritance in an object-oriented data model. Memoranda Informatica 90-77, University of Twente, Enschede, The Netherlands (1990).

[5] C. Batini and M. Lenzerini. A methodology for data schema integration in the ER model. IEEE Transactions on Software Engineering, pp. 650-664 (1984).

[6] C. Batini, M. Lenzerini, and S. Navathe. A comparative analysis of methodologies for database schema integration. ACM Computing Surveys, 18(4):323-364 (1986).

[7] E. Casais. An incremental class rearganization approach. In European Conf. on Object-Oriented Programming, pp. 114-132 (1992)

[8] R. Elmasri and G. Wiederhold. Data model integration using the structural model. In Proc. Int. Conf. on Management of Data, pp. 191-202 (1979).

[9] P. Fankhauser, M. Kracker, and E. Neuhold. Semantic vs. structural resemblance of classes. ACM SIGMOD Record, 20(4):59-63 (1991).

[10] J.-L. Hainaut. Entity-generating schema transformations for entity-relationship models. In Proc. Int. Conf. on the Entity-Relationship Approach (1991).

[11] P. Johannesson. Schema transformations as an aid in view integration. In Proc. Int. Conf. on Advanced Information Systems Engineering, LNCS 685, pp. 71-92. Springer-Verlag, Berlin (1993).

[12] M. Kersten. Goblin: a DBPL designed for advanced database applications. In Proc. Int. Conf. on Database and Expert Systems Applications, pp. 345-349. Springer-Verlag, Wien (1991).

[13] J. Larson, S. Navathe, and R. Elmasri. A theory of attribute equivalence in databases with application to schema integration. IEEE Transactions on Software Engineering, 15(4):449-463 (1989).

[14] C. Lécluse and $\mathrm{P}$. Richard. The $\mathrm{O}_{2}$ database programming language. In Proc. Int. Conf. on Very Large Databases, pp. 411-422. Morgan Kaufmann, Palo Alto, CA (1989).

[15] M. Mannino, S. Navathe, and W. Effelsberg. A rule based approach for merging generalisation hierarchies. Information Systems, 13(3):257-272 (1988).

[16] A. Motro and P. Buneman. Constructing superviews. In Proc. Int. Conf. on Management of Data, PP. 56-64 (1981).

[17] S. Navathe and S. Gadgil. A methodology for view integration in logical data base design. In Proc. Int. Conf. on Very Large Databases, pp. 142-155 (1982).

[18] A. Sheth and S. Gala. Attribute relationships: an impediment in automating schema integration. In Proc. Workshop on Heterogeneous Database Systems (1989).

[19] M. Siegel and S. Madnick. A metadata approach to resolving semantic conflicts. In Proc. International Conference on Very Large Databases, pp. 133-145 (1991).

[20] C. Thieme and A. Siebes. Schema integration in object-oriented databases. In Proc. Int. Conf. on Advanced Information Systems Engineering, LNCS 685, pp. 54-70. Springer-Verlag, Berlin (1993).

[21] C. Thieme and A. Siebes. Schema refinement and schema integration in object-oriented databases. In Proc. Computing Science in The Netherlands, ISBN 906196430 X, pp. 343-354. Stichting Mathematisch Centrum (1993).

[22] C. Thieme and A. Siebes. An approach to schema integration based on transformations and behaviour. Report CS-R9403, CWI, Amsterdam, The Netherlands (1994 (available by anonymous ftp from ftp.cwi.nl)).

[23] C. Yu, W. Sun, S. Dao, and D. Keirsey. Determining relationships among attributes for interoperability of multi-database systems. In Proc. Int. Workshop on Interoperability in Multidatabase Systems, pp. 251-257 (1991). 